\title{
Analyticity of the Pressure for Heisenberg and Plane Rotator Models
}

\author{
François Dunlop \\ Institut des Hautes Etudes Scientifiques, F-91440 Bures-sur-Yvette, France
}

\begin{abstract}
We use complex rotations and convex completion to extend the analyticity region of the pressure for the Heisenberg and plane rotator models.
\end{abstract}

\section{Introduction}

We consider partition functions for 2 or 3 component ferromagnets in a complex external field $\left(\mathbf{H}_{j}\right)_{j=1, \ldots, N}$, where $N$ is the number of sites (eventually $N \rightarrow \infty$ ). Previous results $[1,4]$ on the location of zeros are unsatisfactory, either because the region which is free of zeros has only $N$ complex dimensions (instead of $2 N$ or $3 N$ according to the number of components), or because the real points inside this region are too restricted (external fields in a quadrant instead of a half space). An exception is Fröhlich's result [3] which however relies on the analyticity for large external fields: extra (but standard) assumptions are required, and the resulting region is a very small neighborhood of the real points.

In the first part of this paper, we consider rotation invariant models and extend the analyticity region (for the pressure) from $N$ to $3 N$ complex dimensions by the use of complex rotations (as in axiomatic field theory). If $\tilde{\mathbf{H}}_{j}=\left(H_{j}^{z}, i H_{j}^{x}, i H_{j}^{y}\right)$, the resulting analyticity region contains the forward tube $\left\{\operatorname{Re} \tilde{\mathbf{H}}_{j} \in V_{+}: j=1, \ldots, N\right\}$ together with all its transforms under the real rotations of the original variables.

In the second part, we consider the anisotropic plane rotator model, for which both unsatisfactory results mentioned above are available. Analytic completion again gives analyticity in the forward tube.

\section{Complex Rotation of the Heisenberg Model}

We formulate the result for the quantum $\operatorname{spin} \frac{1}{2}$ Heisenberg model which is more basic: it has a natural Ising spin approximation, and it has many descendents: arbitrary quantum spin, 3 and 2 component classical spins (rotators).

Theorem 1. For $j=1, \ldots, N$, let $S_{j}^{z}, S_{j}^{x}$, $S_{j}^{y}$ denote Pauli matrices acting on the $j$ th factor of the tensor product $\bigotimes_{j=1}^{N} \mathbb{C}^{2}$, and let $\mathbf{H}_{j}=\left(H_{j}^{z}, H_{j}^{x}, H_{j}^{y}\right) \in \mathbb{C}^{3}$. Given positive 
numbers $\left\{J_{i j} \geqq 0: i, j=1, \ldots, N\right\}$, consider the partition function

$$
Z\left(\left(\mathbf{H}_{j}\right)_{j=1, \ldots, N}\right)=\operatorname{Tr} \exp \left\{\sum_{i, j=1}^{N} J_{i j} \mathbf{S}_{i} \cdot \mathbf{S}_{j}+\sum_{i=1}^{N} \mathbf{H}_{i} \cdot \mathbf{S}_{i}\right\} .
$$

Then, for any real unit vector $\mathbf{u}$, the partition function does not vanish in the region

$$
\operatorname{Re}_{j} \cdot \mathbf{u}>\left|\operatorname{Im} \mathbf{H}_{j} \times \mathbf{u}\right|: j=1, \ldots, N .
$$

Proof. By rotation invariance, it is enough to consider $\mathbf{u}$ along the positive $z$ axis. Letting $\tilde{\mathbf{H}}_{j}=\left(H_{j}^{z}, i H_{j}^{x}, i H_{j}^{y}\right)$, the desired region reads

$$
\operatorname{Re} \tilde{\mathbf{H}}_{j} \in V_{+}: j=1, \ldots, N \text {, }
$$

where $V_{+}$is the open forward cone:

$$
V_{+}=\left\{\left(v^{z}, v^{x}, v^{y}\right) \in \mathbb{R}^{3} \mid v^{z^{2}}-v^{x^{2}}-v^{y^{2}}>0\right\} .
$$

Our starting point is the theorem proven by Suzuki and Fisher, extending a previous result of Asano: with our hypotheses (and more generally: see Theorem 2 below), $Z\left(\left(\mathbf{H}_{j}\right)_{j=1, \ldots, N}\right)$ does not vanish when $\operatorname{Re} \tilde{\mathbf{H}}_{j}$ is along the positive $z$ axis for all $j$. Let then $\Lambda$ be a complex rotation $Z\left(\left(\Lambda \mathbf{H}_{j}\right)_{j=1, \ldots, N}\right)$ is an analytic function of the Cayley parameters of $\Lambda$, which is constant for real rotations and therefore also for complex rotations. In particular, we may apply a real Lorentz transformation to $\left(\tilde{\mathbf{H}}_{j}\right)_{j=1, \ldots, N}$, so that $Z\left(\left(\mathbf{H}_{j}\right)_{j}\right)$ does not vanish when $\operatorname{Re} \tilde{\mathbf{H}}_{j}$ is along any given direction $\mathbf{v}$ in $V_{+}$, the same for all $j$. The pressure $N^{-1} \log Z\left(\left(\mathbf{H}_{j}\right)_{j}\right)$ is then analytic in a neighborhood of a tube:

$$
\operatorname{Re}\left(\tilde{\mathbf{H}}_{j}\right)_{j=1, \ldots, N} \in \bigcup_{\mathbf{v} \in V_{+}}\left\{\left(\lambda_{j} \mathbf{v}\right)_{j=1, \ldots, N} \mid \lambda_{j}>0: j=1, \ldots, N\right\} .
$$

In order to apply the (flat) tube theorem, we verify that the basis (4) may be connected by piecewise linear curves: $\left(\lambda_{j} \mathbf{v}\right)_{j=1, \ldots, N}$ and $\left(\lambda_{j}^{\prime} \mathbf{v}^{\prime}\right)_{j=1, \ldots, N}$ are endpoints ( $t=0$ and $t=2$ respectively) of:

$$
\left(\lambda_{j}(t) \mathbf{v}(t)\right)_{j=1, \ldots, N}=\left\{\begin{array}{l}
\left(\lambda_{j}\left(t \mathbf{v}^{\prime}+(1-t) \mathbf{v}\right)\right)_{j=1, \ldots, N}: 0 \leqq t \leqq 1 \\
\left(\left((2-t) \lambda_{j}+(t-1) \lambda_{j}^{\prime}\right) \mathbf{v}^{\prime}\right)_{j=1, \ldots, N} 1 \leqq t \leqq 2
\end{array}\right.
$$

which lies inside (4). It follows that the pressure extends analytically to the convex hull of this tube, i.e., to (3). Indeed, given a point $\left(\tilde{\mathbf{H}}_{j}\right)_{j=1, \ldots, N}$ in (3), there will exist $\varepsilon$ such that

$$
\mathbf{v}^{(k)}=\tilde{\mathbf{H}}_{k}-\varepsilon \sum_{j \neq k} \tilde{\mathbf{H}}_{j} \in V_{+}+i \mathbb{R}^{3}: k=1, \ldots, N
$$

and

$$
\lambda_{j}^{(k)}=\varepsilon+(1-\varepsilon(N-1)) \delta_{j, k}>0: j, k=1, \ldots, N .
$$

The sum

$$
\sum_{k=1}^{N}\left(\lambda_{j}^{(k)} \mathbf{v}^{(k)}\right)_{j=1, \ldots, N}
$$


is then proportional to $\left(\tilde{\mathbf{H}}_{j}\right)_{j=1, \ldots, N}$ and the desired result follows from the scale invariance of the domain. This concludes the proof of Theorem 1.

As we shall see in the next section, rotation invariance is probably not necessary for the pressure to be analytic in some fixed forward tube in $\left(\tilde{\mathbf{H}}_{j}\right)_{j}$, with a suitable choice of the $z$ axis. In order to discuss briefly the anisotropic Heisenberg model, we need to state the theorem of Suzuki and Fisher in its generality (for quantum $\left.\operatorname{spin} \frac{1}{2}\right)$ :

Theorem 2 [4]. Let $\left\{J_{i j}\right\}_{i, j=1, \ldots, N}$ be $3 \times 3$ matrices such that $J_{i j}^{z z}, J_{i j}^{x x}, J_{i j}^{y y}, J_{i j}^{x y}, J_{i j}^{y x}$ are real and $J_{i j}^{z x}, J_{i j}^{x z}, J_{i j}^{z y}, J_{i j}^{y z}$ are pure imaginary. Suppose that, for all $i, j$

$$
\begin{aligned}
2 J_{i j}^{z z} \geqq & \left(\left(J_{i j}^{x x}-J_{i j}^{y y}\right)^{2}+\left(J_{i j}^{x y}+J_{i j}^{y x}\right)^{2}\right)^{1 / 2}+\left(\left(J_{i j}^{x x}+J_{i j}^{y y}\right)^{2}+\left(J_{i j}^{x y}-J_{i j}^{y x}\right)^{2}\right)^{1 / 2} \\
& +2\left(\left|J_{i j}^{x z}\right|^{2}+\left|J_{i j}^{y z}\right|^{2}\right)^{1 / 2}+2\left(\left|J_{i j}^{z x}\right|^{2}+\left|J_{i j}^{z y}\right|^{2}\right)^{1 / 2} .
\end{aligned}
$$

Then the partition function,

$$
Z\left(\left(\mathbf{H}_{j}\right)_{j=1, \ldots, N}\right)=\operatorname{Tr} \exp \left\{\sum_{i, j=1}^{N} \mathbf{S}_{i} \cdot J_{i j} \mathbf{S}_{j}+\sum_{i=1}^{N} \mathbf{H}_{i} \cdot \mathbf{S}_{i}\right\}
$$

does not vanish when

$$
\left\{\begin{array}{l}
\operatorname{Re} H_{j}^{z}>0: j=1, \ldots, N \\
H_{j}^{x}, H_{j}^{y} \text { real }: j=1, \ldots, N .
\end{array}\right.
$$

Notice that the reality conditions in the hypotheses of Theorem 2 are stable under the Lorentz transformations used in the proof of Theorem 1 (i.e., under $J_{i j} \mapsto \Lambda J_{i j} \Lambda^{T}$ ). On the other hand, the bounds (8) will restrict the applicability of the previous method. For the sake of simplicity, we shall assume $J_{i j}^{x y}=J_{i j}^{y x}=0$.

Theorem 3. Let $\left\{J_{i j}\right\}_{i, j=1, \ldots, N}$ be $3 \times 3$ diagonal matrices such that

$$
J_{i j}^{z z} \geqq\left|J_{i j}^{x x}\right|: i, j=1, \ldots, N
$$

and

$$
J_{i j}^{z z} \geqq\left|J_{i j}^{y y}\right|: i, j=1, \ldots, N .
$$

Let

$$
\begin{aligned}
& \alpha_{x}=\inf _{i, j} \sup _{\lambda}\left\{\operatorname{th} \lambda \mid J_{i j}^{z z}+J_{i j}^{x x} \geqq\left(J_{i j}^{z z}-J_{i j}^{x x}\right) \operatorname{Sh} 2 \lambda \text { and } J_{i j}^{z z}-\left|J_{i j}^{y y}\right| \geqq\left(J_{i j}^{z z}-J_{i j}^{x x}\right) \operatorname{Sh} 2 \lambda\right\} \\
& \alpha_{y}=\inf _{i, j} \sup _{\lambda}\left\{\operatorname{th} \lambda \mid J_{i j}^{z z}+J_{i j}^{y y} \geqq\left(J_{i j}^{z z}-J_{i j}^{y y}\right) \operatorname{Sh} 2 \lambda \text { and } J_{i j}^{z z}-\left|J_{i j}^{x x}\right| \geqq\left(J_{i j}^{z z}-J_{i j}^{y y}\right) \operatorname{Sh} 2 \lambda\right\} .
\end{aligned}
$$

Then the partition function (9) does not vanish when

$$
\frac{\left|\operatorname{Im} H_{j}^{x}\right|}{\alpha_{x}}+\frac{\left|\operatorname{Im} H_{j}^{y}\right|}{\alpha_{y}}<\operatorname{Re} H_{j}^{z}: j=1, \ldots, n .
$$

Proof. The idea is to use Lorentz covariance to bring the external field from (12) back to (10). The attention will be concentrated on the couplings $J_{i j}$, which we now write explicitly as arguments of the partition function $Z\left(\left\{J_{i j}\right\}_{i, j=1, \ldots, N}\right.$ : $\left.\left(\mathbf{H}_{j}\right)_{j=1, \ldots, N}\right)$ defined in (9). For any complex rotation $\Lambda$, analytic continuation from 
the real rotations implies

$$
Z\left(\left\{J_{i j}\right\}_{i, j=1, \ldots, N} ;\left(\Lambda \mathbf{H}_{j}\right)_{j=1, \ldots, N}\right)=Z\left(\left\{\Lambda J_{i j} \Lambda^{T}\right\}_{i, j=1, \ldots, N} ;\left(\mathbf{H}_{j}\right)_{j=1, \ldots, N}\right) .
$$

Let us assume $J_{i j}$ diagonal and $\operatorname{Re} \tilde{\mathbf{H}}_{j}$ along the positive $z$ axis, for all $i, j$. We first take for $\Lambda$ a Lorentz rotation in the $z, x$ plane. Dropping the indices $i, j$, we have:

$$
\Lambda J_{i j} \Lambda^{T}=\left(\begin{array}{ccc}
\frac{J^{z z}-J^{x x}}{2} \mathrm{Ch} 2 \lambda+\frac{\mathrm{J}^{z z}+\mathrm{J}^{x x}}{2} & -i \frac{J^{z z}-J^{x x}}{2} \operatorname{Sh} 2 \lambda & 0 \\
-i \frac{J^{z z}-J^{x x}}{2} \operatorname{Sh} 2 \lambda & -\frac{J^{z z}-J^{x x}}{2} \mathrm{Ch} 2 \lambda+\frac{J^{z z}+J^{x x}}{2} & 0 \\
0 & 0 & J^{y y}
\end{array}\right)
$$

The right hand side of (13) will satisfy Theorem 2 if and only if:

$$
\left\{\begin{array}{l}
\left(J^{z z}-J^{x x}\right) \operatorname{Ch} 2 \lambda+J^{z z}+J^{x x} \geqq\left|-\left(J^{z z}-J^{x x}\right) \operatorname{Ch} 2 \lambda+J^{z z}+J^{x x}\right|+2\left|J^{z z}-J^{x x}\right||\operatorname{Sh} 2 \lambda| \\
\left(J^{z z}-J^{x x}\right) \operatorname{Ch} 2 \lambda+J^{z z}+J^{x x} \geqq 2\left|J^{y y}\right|+2\left|J^{z z}-J^{x x}\right||\operatorname{Sh} 2 \lambda|
\end{array}\right.
$$

which we replace by the stronger conditions:

$$
\left\{\begin{array}{l}
J^{z z}+J^{x x} \geqq\left(J^{z z}-J^{x x}\right)|\operatorname{Sh} 2 \lambda| \geqq 0 \\
J^{z z}-\left|J^{y y}\right| \geqq\left(J^{z z}-J^{x x}\right)|\operatorname{Sh} 2 \lambda|
\end{array}\right.
$$

Comparing with (11) we see that the pressure will be analytic in a neighborhood of the tube

$$
\bigcup_{|\alpha|<\alpha_{x}}\left\{\left(\tilde{\mathbf{H}}_{j}\right)_{j=1, \ldots, N} \mid \operatorname{Re} \tilde{\mathbf{H}}_{j}^{x}=\alpha \operatorname{Re} \tilde{\mathbf{H}}_{j}^{z} \text { and } \operatorname{Re} \tilde{\mathbf{H}}_{j}^{y}=0: j=1, \ldots, N\right\} .
$$

The union of this set with the set obtained by exchanging the roles of $x$ and $y$ is

$$
\bigcup_{\mathbf{v} \in C}\left\{\left(\tilde{\mathbf{H}}_{j}\right)_{j=1, \ldots, N} \mid \operatorname{Re} \tilde{\mathbf{H}}_{j}=\lambda_{j} \mathbf{v}, \lambda_{j}>0: j=1, \ldots, N\right\},
$$

where

$$
C=\left\{\mathbf{v} \in \mathbb{R}^{3}|| v^{x} \mid<\alpha_{x} v^{z} \text { and } v^{y}=0\right\} \cup\left\{\mathbf{v} \in \mathbb{R}^{3}|| v^{y} \mid<\alpha_{y} v^{z} \text { and } v^{x}=0\right\} .
$$

The same reasoning as in the proof of Theorem 1 shows that the convex hull of (18) is given by

$$
\bigcup_{\mathbf{v} \in \hat{C}}\left\{\left(\tilde{\mathbf{H}}_{j}\right)_{j=1, \ldots, N} \mid \operatorname{Re} \tilde{\mathbf{H}}_{j} \in \hat{C}: j=1, \ldots, N\right\} .
$$

$\hat{C}$ being the convex hull of $C$ :

$$
\hat{C}=\left\{\mathbf{v} \in \mathbb{R}^{3} \mid \frac{\left|v^{x}\right|}{\alpha_{x}}+\frac{\left|v^{y}\right|}{\alpha_{y}}<v^{z}\right\} .
$$

This concludes the proof of Theorem 3 . 


\section{Anisotropic Plane Rotators}

We shall now consider two-component "classical spins", for which a non zero lowerbound on the modulus of the complex partition function is available by the method of correlation inequalities:

Theorem 4 [1]. Given $2 \times 2$ matrices $\left\{J_{i j}\right\}_{i, j=1, \ldots, N}$ such that $J_{i j}^{x x}, J_{i j}^{y y}$ are real, $J_{i j}^{x y}, J_{i j}^{y x}$ are pure imaginary $(i, j=1, \ldots, N)$ and

$$
\begin{aligned}
& J_{i j}^{x x}+J_{i j}^{y y} \geqq\left|J_{i j}^{x y}-J_{i j}^{y x}\right| \\
& J_{i j}^{x x}-J_{i j}^{y y} \geqq\left|J_{i j}^{x y}+J_{i j}^{y x}\right|
\end{aligned} \quad i, j=1, \ldots, N .
$$

Let $\mathbf{H}_{j}=\left(H_{j}^{x}, H_{j}^{y}\right) \in \mathbb{C}^{2}, j=1, \ldots, N$ and

$$
Z\left(\left(\mathbf{H}_{j}\right)_{j=1, \ldots) N}\right)=\int \prod_{j=1}^{N}\left(\delta\left(\mathbf{S}_{j}^{2}-1\right) d^{2} \mathbf{S}_{j}\right) \exp \left\{\sum_{i, j=1}^{N} \mathbf{S}_{i} \cdot J_{i j} \mathbf{S}_{j}+\sum_{j=1}^{N} \mathbf{H}_{j} \cdot \mathbf{S}_{j}\right\} .
$$

Suppose that

$$
\begin{aligned}
& \operatorname{Re}\left(H_{j}^{x}+i H_{j}^{y}\right) \geqq\left|\operatorname{Im}\left(H_{j}^{x}+i H_{j}^{y}\right)\right| \\
& \operatorname{Re}\left(H_{j}^{x}-i H_{j}^{y}\right) \geqq\left|\operatorname{Im}\left(H_{j}^{x}-i H_{j}^{y}\right)\right|
\end{aligned} \quad j=1, \ldots, N .
$$

Then

$$
\left|Z\left(\left(\mathbf{H}_{j}\right)_{j=1, \ldots, N}\right)\right| \geqq|Z(0)|>0 .
$$

Remark. Imaginary couplings were not considered in [1], but the conditions (20) fit easily in the framework.

Our aim is to make an analytic completion of the union of the domains given by Theorem 4 and by the analog of Theorem 2 for plane rotators:

Theorem 5 [2]. Given $2 \times 2$ matrices $\left\{J_{i j}\right\}_{i, j=1, \ldots, N}$ such that $J_{i j}^{x x}, J_{i j}^{y y}$ are real, $J_{i j}^{x y}, J_{i j}^{y x}$ are pure imaginary and

$$
J_{i j}^{x x} \geqq\left|J_{i j}^{y y}\right|+\left|J_{i j}^{x y}\right|+\left|J_{i j}^{y x}\right|: i, j=1, \ldots, N .
$$

Suppose that

$$
\begin{aligned}
& \operatorname{Re} H_{j}^{x}>0 \\
& \operatorname{Im} H_{j}^{y}=0
\end{aligned} \quad j=1, \ldots, N .
$$

Then the partition function (21) is different from zero.

Notice that the real points satisfying (25) may vary in a half plane, whereas (22) allows only a quadrant $\left(\operatorname{Re} H_{j}^{x} \geqq\left|H_{j}^{y}\right|\right)$. On the other hand, (25) would imply that the pressure is analytic in $\left(H_{j}^{x}\right)_{j=1, \ldots, N}$ but not necessarily in $\left(H_{j}^{y}\right)_{j=1, \ldots, N}$. Our result is the following:

Theorem 6. Given $2 \times 2$ matrices $\left\{J_{i j}\right\}_{i, j=1, \ldots, N}$ such that $J_{i j}^{x x}, J_{i j}^{y y}$ are real, $J_{i j}^{x y}, J_{i j}^{y x}$ are pure imaginary and

$$
\begin{aligned}
& J_{i j}^{x x}+J_{i j}^{y y} \geqq\left|J_{i j}^{x y}-J_{i j}^{y x}\right| \\
& J_{i j}^{x x}-J_{i j}^{y y} \geqq\left|J_{i j}^{x y}+J_{i j}^{y x}\right|
\end{aligned} \quad i, j=1, \ldots, N .
$$


Suppose that

$$
\begin{aligned}
& \operatorname{Re}\left(H_{j}^{x}+i H_{j}^{y}\right) \geqq 0 \\
& \operatorname{Re}\left(H_{j}^{x}-i H_{j}^{y}\right) \geqq 0
\end{aligned} \quad j=1, \ldots, N .
$$

Then the partition function (21) is different from zero.

Remark. If we write the interaction $\mathbf{S}_{i} \cdot J_{i j} \mathbf{S}_{j}$ in terms of the "light cone" variables $\left(S_{j}^{x} \pm i S_{j}^{y}\right)_{j=1, \ldots, N}$, then the matrix $J_{i j}$ is transformed into

$$
\tilde{J}_{i j}=\left(\begin{array}{ll}
a & b \\
c & d
\end{array}\right)
$$

and (26) is equivalent to $a, b, c, d$ being positive. But (24) requires in addition

$$
\left\{\begin{array}{l}
a+d \geqq|b-c| \\
b+c \geqq|a-d| .
\end{array}\right.
$$

We shall prove the theorem, assuming (24) [or (28)]. Using the Lorentz invariance of the domain (27), we shall then recover the whole of (26). Indeed

$$
\tilde{\Lambda} \tilde{J}_{i j} \tilde{\Lambda}^{T}=\left(\begin{array}{cc}
a \lambda^{2} & b \\
c & d \lambda^{-2}
\end{array}\right)
$$

and for arbitrary $a, b, c, d$ positive, $a+d \neq 0$, there will exist $\lambda>0$ such that

$$
\left\{\begin{array}{l}
a \lambda^{2}+d \lambda^{-2} \geqq|b-c| \\
b+c \geqq\left|a \lambda^{2}-d \lambda^{-2}\right| .
\end{array}\right.
$$

The limit points $a=d=0$ are included by uniform convergence.

The proof of Theorem 6 is now a consequence of the following lemma:

Lemma. Let $f\left(\left(\mathbf{H}_{j}\right)_{j=1, \ldots, N}\right)$ be analytic in a neighborhood of the following two regions
(I) $\left\{\begin{array}{l}\operatorname{Re} H_{j}^{x}>0 \\ \operatorname{Im} H_{j}^{y}=0\end{array} \quad j=1, \ldots, N\right.$;
(II) $\left\{\begin{array}{l}\operatorname{Im} H_{j}^{x}=0, \operatorname{Re} H_{j}^{x}>a_{0} \\ \left|\operatorname{Re} H_{j}^{y}\right|+\left|\operatorname{Im} H_{j}^{y}\right|<\alpha\left(H_{j}^{x}-a_{0}\right)\end{array} \quad j=1, \ldots, N\right.$.

Then $f\left(\left(\mathbf{H}_{j}\right)_{j=1, \ldots, N}\right)$ extends analytically to the tube

$$
\left|\operatorname{Im} H_{j}^{y}\right|<\alpha \operatorname{Re} H_{j}^{x}: j=1, \ldots, N .
$$

Remarks. The logarithm of the partition function (21) with the restriction (24) satisfies the lemma with $\alpha=1, a_{0}=0$.

We can add more components satisfying the same conditions as $\left(H_{j}^{y}\right)_{j=1, \ldots, N}$ : the lemma and its proof extend trivially.

Large external field expansions may be used in place of Theorem 4 to obtain the region (II). Then $\alpha$ is small and $a_{0}$ is large. This is the way Fröhlich [3] proved analytically in some (unspecified) complex neighborhood of $\left\{H_{j}^{x}>0: j=1, \ldots, N\right\}$. Proof of the Lemma. The region (II) is the union over $\left(a_{j}\right)_{j=1, \ldots, N}$ of the topological products

(IIa) $\left\{\begin{array}{l}H_{j}^{x}>a_{j} \\ \left|\operatorname{Re} H_{j}^{y}\right|+\left|\operatorname{Im} H_{j}^{y}\right|<\alpha\left(a_{j}-a_{0}\right)\end{array} \quad j=1, \ldots, N\right.$. 
In order to apply the tube theorem to the region (I) $\cup(\mathrm{IIa})$, we introduce the following variables:

$$
\begin{aligned}
\varphi_{j} & =\operatorname{Arg} \operatorname{Ch} \frac{H_{j}^{x}}{a_{j}} \\
\psi_{j} & =\log \frac{b_{j}+H_{j}^{y}}{b_{j}-H_{j}^{y}}, \quad b_{j}=\alpha\left(a_{j}-a_{0}\right) .
\end{aligned}
$$

In these new variables, we have a function analytic in a neighborhood of the following two tubes:

$\left(\mathrm{I}^{\prime}\right)\left\{\begin{array}{l}0 \leqq \operatorname{Im} \varphi_{j}<\frac{\pi}{2} \\ \operatorname{Im} \psi_{j}=0\end{array} \quad j=1, \ldots, N ; \quad\left(\mathrm{II}^{\prime} \mathrm{a}\right)\left\{\begin{array}{l}\operatorname{Im} \varphi_{j}=0 \\ -\frac{\pi}{4}<\operatorname{Im} \psi_{j}<\frac{\pi}{4}\end{array} \quad j=1, \ldots, N\right.\right.$.

Therefore it extends analytically to the union over $t, 0 \leqq t \leqq 1$, of the regions

$$
\left\{\begin{array}{l}
0 \leqq \operatorname{Im} \varphi_{j}<(1-t) \frac{\pi}{2} \\
-t \frac{\pi}{4}<\operatorname{Im} \psi_{j}<\mathrm{t} \frac{\pi}{4}
\end{array} \quad j=1, \ldots, N .\right.
$$

Consider now a point $\left(\mathbf{H}_{j}\right)_{j=1, \ldots, N}$ in the original variables, such that

$$
\left|\operatorname{Im} H_{j}^{y}\right|<\frac{\alpha}{4} \operatorname{Re} H_{j}^{x}: j=1, \ldots, N \text {. }
$$

Its image in the variables $\left(\varphi_{j}, \psi_{j}\right)_{j=1, \ldots, N}$ will be in (33) for sufficiently small $t$ and suitable large $a_{j}$ 's. Indeed, as $a_{j} \rightarrow \infty$,

$$
\left\{\begin{array}{l}
\frac{\pi}{2}-\operatorname{Im} \varphi_{j} \sim \frac{\operatorname{Re} H_{j}^{x}}{a_{j}} \\
\operatorname{Im} \psi_{j} \sim 2 \frac{\operatorname{Im} H_{j}^{y}}{b_{j}}
\end{array}\right.
$$

so that we should take $a_{j} \approx t^{-1}$ with

$$
\frac{8\left|\operatorname{Im} H_{j}^{y}\right|}{\alpha \pi}<a_{j} t<\frac{2 \operatorname{Re} H_{j}^{x}}{\pi} .
$$

This concludes the first step of the proof: analyticity in the region (34). Note that the result does not depend on the value of $a_{0}$. An iteration of this first step will now eliminate the factor $\frac{1}{4}$ in (34). To make the procedure more clear, we start from an even weaker form of the lemma: with the same hypotheses, there should exist $\theta>0$ such that $f$ extends analytically to

$$
\left|\operatorname{Im} H_{j}^{y}\right|<\theta \alpha \operatorname{Re} H_{j}^{x}: j=1, \ldots, N .
$$

Let then

$$
H_{j}^{y, 1}=H_{j}^{y}-i \theta_{1} \alpha H_{j}^{x}
$$

with $-\theta<\theta_{1}<\theta$. 
In the variables $\left(H_{j}^{x}\right)_{j=1, \ldots, N}$ and $\left(H_{j}^{y, 1}\right)_{j=1, \ldots, N}$, one sees from (37) and (II) that $f$ is analytic in a neighborhood of

$\left(\mathrm{I}^{(1)}\right) \quad\left\{\begin{array}{l}\operatorname{Re} H_{j}^{x}>0 \\ \operatorname{Im} H_{j}^{y, 1}=0\end{array} \quad j=1, \ldots, N\right.$

$\left(\mathrm{II}^{(1)}\right)\left\{\begin{array}{l}\operatorname{Im} H_{j}^{x}=0, H_{j}^{x}>\frac{a_{0}}{1-\theta} j=1, \ldots, N ; \\ \left|\operatorname{Re} H_{j}^{y, 1}\right|+\left|\operatorname{Im} H_{j}^{y, 1}\right|<(1-\theta) \alpha\left(H_{j}^{x}-\frac{a_{0}}{1-\theta}\right)\end{array}\right.$

so that applying our first step gives analyticity in

$$
\left|\operatorname{Im} H_{j}^{y, 1}\right|<(1-\theta) \theta \alpha \operatorname{Re} H_{j}^{x} \quad j=1, \ldots, N .
$$

We proceed inductively with

$$
\left\{\begin{array}{l}
H_{j}^{y, p}=H_{j}^{y, p-1}-i \theta_{p} \alpha H_{j}^{x} \\
-\theta(1-\theta)^{p-1}<\theta_{p}<\theta(1-\theta)^{p-1} \quad j=1, \ldots, N
\end{array}\right.
$$

and obtain analyticity in

$$
\left|\operatorname{Im} H_{j}^{y, p}\right|<(1-\theta)^{p} \theta \alpha \operatorname{Re} H_{j}^{x} \quad j=1, \ldots, N .
$$

If we now vary $\theta_{1}, \theta_{2}, \ldots$, in the allowed intervals, the resulting analyticity region for the original variables reads:

$$
\left|\operatorname{Im} H_{j}^{y}\right|<\left(\sum_{p=0}^{\infty}(1-\theta)^{p}\right) \theta \alpha \operatorname{Re} H_{j}^{x}=\alpha \operatorname{Re} H_{j}^{x}: j=1, \ldots, N .
$$

This concludes the proof of the lemma.

Acknowledgements. We are indebted to D. Ruelle for his suggestion to apply "Lorentz transformations" to the isotropic Heisenberg model, and to H. Epstein for private lectures on convex analytic completion, as well as for his idea to iterate the weaker form of our lemma.

\section{References}

1. Dunlop, F.: Zeros of the partition function and gaussian inequalities for the plane rotator model. J. Stat. Phys. 21 ; No. 5 (1979)

2. Dunlop, F., Newman, C.M. : Multicomponent field theories and classical rotators. Commun. Math. Phys. 44, 223-235 (1975)

3. Fröhlich, J.: Poetic phenomena in (two dimensional) quantum field theory. In: Les méthodes mathématiques de la théorie quantique des champs. Paris : C.N.R.S. 1976

4. Suzuki, M., Fisher, M. : Zeros of the partition function for the Heisenberg, ferro electric, and general Ising models. J. Math. Phys. 12, 235-246 (1971)

Communicated by E. Lieb 